\title{
Initial Diagnostic Errors in Children Suspected of Having Heart Disease. Prevalence and Long-Term Consequences
}

\author{
Fernando Amaral, João A. Granzotti \\ Ribeirão Preto, SP - Brazil
}

\begin{abstract}
Objective-To access the incidence of diagnostic errors in the initial evaluation of children with cardiac murmurs.
\end{abstract}

Methods - We evaluated our 7-years of experience in a public pediatric cardiology outpatient clinic. Of 3692 patients who were sent to the hospital, 2603 presented with a heart murmur and were investigated. Patients for whom a disagreement existed between the initial and final diagnoses were divided into the following 2 groups: G1 $(n=17)$ with an initial diagnosis of an innocent murmur and a final diagnosis of cardiopathy, and G2 $(n=161)$ with an initial diagnosis of cardiopathy and a final diagnosis of a normal heart.

Results - In G1, the great majority of patients had cardiac defects with mild hemodynamic repercussions, such as small ventricular septal defect and mild pulmonary stenosis. In G2, the great majority of structural defects were interventricular communication, atrial septal defect and pulmonary valve stenosis.

Conclusion - A global analysis demonstrated that diagnostic error in the initial evaluation of children with cardiac murmurs is real, reaching approximately $6 \%$ of cases. The majority of these misdiagnoses were in patients with an initial diagnosis of cardiopathy, which was not confirmed through later complementary examinations. Clinical cardiovascular examination is an excellent resource in the evaluation of children suspected of having cardiopathy. Immediate outpatient discharge of children with an initial diagnosis of an innocent heart murmur seems to be a suitable approach.

Key words: congenital cardiopathy, murmur, children

Faculdade de Medicina de Ribeirão Preto, USP, Hospital do Coração de Ribeirão Preto - Fundação Waldemar B. Pessoa e Faculdade de Medicina da UNAERP. Mailing address: Fernando Amaral - Av. Independência, 1387 - 14025-390 Ribeirão Preto, SP - Brazil - E-mail: ftvamaral@bol.com.br
A considerable number of children evaluated at a daily care ambulatory clinic were sent to a pediatric cardiological ambulatory clinic to be evaluated for congenital cardiopathy. As already demonstrated, most patients are considered normal after a suitable evaluation. However, a small number of patients have cardiopathy, generally with lower hemodynamic repercussions ${ }^{1-3}$. These numbers tend to be different at an experienced center where the incidence of congenital cardiopathy is usually very high ${ }^{4}$. The initial evaluation of these patients is usually performed by the general pediatrician, and a heart murmur is the most frequent clinical finding ${ }^{2,3,5}$. These cases are then examined by the pediatric cardiologist, and the clinical examination alone frequently enables the differential diagnosis between an innocent murmur and a pathological murmur, with no need for complementary tests ${ }^{1,6}$.

With the purpose of assessing the level of clinical accuracy in the characterization of a murmur as innocent or pathological, clinical investigations ${ }^{1}$ have demonstrated that the rate of agreement between the initial diagnosis (clinical) and the final diagnosis (clinical + laboratory tests) may reach approximately $90 \%$, demonstrating the high level of sensitivity of the physical examination performed by an experienced pediatric cardiologist. Despite this high index of diagnostic agreement, initial and final diagnoses may differ in approximately $10 \%$ of the cases. This may lead to potentially unfavorable consequences for these children, because we have discharged patients with an initial diagnosis of innocent murmur without performing complementary tests, apart from an electrocardiogram. Even if we consider that the errors in the initial evaluation of these patients demonstrates mild forms of cardiopathy, the results reported are based on experience with a small number of patients ${ }^{1,5,7}$, including ours ${ }^{6}$, when we studied the importance of noninvasive tests in children with a heart murmur. With the purpose of verifying the incidence of diagnostic errors in the initial evaluation of children with a heart murmur, we reviewed our experience of 7 years in a public ambulatory pediatric cardiology clinic in Ribeirão Preto. 


\section{Methods}

Between September 1990 and August 1997, 3692 children, initially examined in the Unidades Básicas de Saúde (Basic Health Units) in the city of Ribeirão Preto, were sent for pediatric cardiologic evaluation. A clinical examination was performed in all patients in the dorsal decubitus position and in a suitable environment, and 2603 patients were sent for more accurate evaluation because of the presence of a heart murmur. All patients underwent electrocardiography. Chest radiography and echocardiography were performed in suspected cases (cases that were not clear on clinical examination) ${ }^{1}$, and for those cases that were clearly pathological (patients with a pathological murmur). As already mentioned ${ }^{2,3,6}$, echocardiography was not a routine examination, and the cases of innocent murmur studied were those sent with a reliable echocardiogram previously obtained. These patients were assessed, and the cases where disagreement between the initial and final diagnosis was detected were divided into the following 2 groups: group 1 (17 patients) with an initial diagnosis of innocent murmur and a final diagnosis of cardiopathy, and group 2 (161 patients) with an initial diagnosis of cardiopathy and a final diagnosis of a normal heart.

\section{Results}

Of the 2603 cases studied, disagreement between the initial and final diagnosis was detected in 178 cases $(6.7 \%)$.

Group 1 (initial diagnosis of innocent murmur and final diagnosis of cardiopathy) comprised 17 patients $(0.7 \%), 9$ females and 8 males. Most patients $(47 \%)$ were of school age, $29 \%$ of preschool age and $24 \%$ were infants. Fourteen patients ( $82 \%$ ) were sent only because of a heart murmur, whereas 3 also had apparently abnormal X-rays, a history of rheumatic fever, and a suspicion of arrhythmia. The final diagnosis of these 17 patients was atrial septal defect(ASD), 4 cases (24\%); mitral regurgitation, 3 cases (17\%); mitral valve prolapse (MVP), 3 cases (17\%); Wolff-ParkinsonWhite syndrome, 2 cases (12\%); and dilated myocardial disease, small ventricular septal defect (VSD), mild pulmonary stenosis, mild aortic regurgitation, and idiopathic dilation of the pulmonary trunk, 1 case (6\%) each.

In 3 cases, cardiopathy was detected based only on laboratory tests, which demonstrated 2 cases of WolffParkinson-White syndrome diagnosed by electrocardiography and 1 case of dilated myocardial disease suspected by X-ray (tab. I)

Group 2 (initial diagnosis of cardiopathy and final diagnosis of normal heart) comprised 161 patients (6.2\%); 102 patients $(63 \%)$ were male and 59 patients $(37 \%)$ were female. Most patients (36\%) were of school age, $32 \%$ were in preschool age, $26 \%$ were infants, and $6 \%$ were neonates. One hundred and thirty-eight patients $(86 \%)$ were sent only because of a heart murmur, whereas 23 other patients also had apparently abnormal chest X-rays, 8 patients $(5 \%)$; precordial pain, 5 patients (3\%); dyspnea, 5 patients (3\%); syncope, 3 patients (2\%); arrhythmia and a history of rheumatic fever, 1 patient $(0.5 \%)$ each.

The initial diagnosis of these 161 patients was VSD, 72 patients (45\%); ASD, 30 patients (19\%); pulmonary valve stenosis, 13 patients $(8 \%)$; aortic valve stenosis, 11 patients (7\%); subaortic stenosis, 10 patients $(6 \%)$; dilated cardiomyopathy, 8 patients (5\%); MVP, 6 patients (4\%); PDA patent ductus arteriosus, 5 patients (3\%); bicuspid aortic valve, and mitral regurgitation, 2 patients ( $1 \%$ ) each; aortic coarctation and tricuspid valve prolapse, 1 patient $(0.5 \%)$ each (tab. II).

\section{Discussion}

It is well known that heart murmurs are the most common cause for children being sent to the ambulatory pediatric cardiology clinic. These murmurs are usually detected during routine pediatric examinations and the majority of them are considered innocent ${ }^{1-3}$. This diagnosis is usually established solely through clinical examination, without the need of laboratory tests, as long as an experien-

\begin{tabular}{|cll|}
\hline \multicolumn{2}{|c|}{$\begin{array}{c}\text { Table I - Semiologic finding in } \mathbf{1 7} \text { cases with initial diagnoses of } \\
\text { innocent murmurs and abnormal complementary tests (G1) }\end{array}$} \\
\hline $\mathrm{N}^{\circ}$ of cases & Semiologic finding & Diagnoses - ECO \\
\hline & SM PA + normal B2 & ASD \\
3 & SM $3^{\circ}$ and $4^{\circ}$ LICS & Mild MR (Doppler) \\
3 & SM $3^{\circ}$ and $4^{\circ}$ LICS & Mitral prolapse \\
2 & SM PA & WPW (ECG) \\
1 & SM PS + abnormal X-ray & Cardiomyopathy \\
1 & SM $3^{\circ}$ and $4^{\circ}$ LICS & Small VSD \\
1 & SM PA & Mild PS \\
1 & SM PA & TAP dilation \\
1 & SM $3^{\circ}$ and $4^{\circ}$ LICS & Mild Ao R (Doppler) \\
\hline SM-systolic murmur; PA-pulmonary site; B2-2nd sound; ASD-atrial septal \\
defect; LICS-left intercostal space; WPW-Wolff - Parkinson - White \\
syndrome; Rx-simple Chest X-ray; VSD-ventricular septal defect; PS- \\
pulmonary stenosis; AoR- aortic regurgitation.
\end{tabular}

\begin{tabular}{|cll|}
\hline \multicolumn{2}{|c|}{$\begin{array}{c}\text { Table II } \text { - Semiologic finding and clinical suspicion of initial } \\
\text { diagnosis of } \mathbf{1 6 1} \text { cases with normal complementary tests (G2) }\end{array}$} \\
\hline $\mathrm{N}^{\mathbf{o}}$ of cases & Semiologic finding & Clinical suspicion \\
\hline 72 & SM $3^{\text {o }}$ and $4^{\text {o }}$ EICE & VSD \\
30 & SM PA & ASD \\
13 & SM PA & PS \\
11 & SM AoF & AoS \\
10 & SM $3^{\circ}$ and $4^{\circ}$ EICE & Subvalvular AoS \\
8 & Abnormal X-ray & Myocardial disease \\
6 & SM Mitral & Mitral prolapse \\
5 & SM FP (neonate) & Patent arterial channel \\
2 & SM Mitral & IM \\
2 & Aortic Click & BAV \\
1 & Femoral Pulse $\Downarrow$ & AoCo \\
1 & Tricuspid SS & Tricuspid prolapse \\
\hline AoF-aortic focus; & AoS-valvar aortic stenosis; BAV-bicuspid aortic valve; \\
$\Downarrow$ decreased amplitude; AoCo-Aortic coarctation; the others are as expressed \\
in table I.
\end{tabular}


ced pediatric cardiologist is involved in making the diagnosis ${ }^{1,5-7}$. Because of these conclusions and mainly because of the high cost of laboratory tests ${ }^{8}$, we routinely discharge patients with innocent murmurs without performing any complementary examinations other than electrocardiography, still routinely performed. However, as we have already mentioned ${ }^{1,7}$, this clinical diagnosis may be mistaken generally for cardiopathies of small clinical repercussion in approximately $10 \%$ of cases. Recently, we had the opportunity of confirming these observations ${ }^{6}$, demonstrating that clinical examination alone may be extremely sensitive but not always absolute in differentiating between innocent and pathologic murmurs. Although this conduct of discharging children with innocent murmurs is based on wellconducted investigations, we should consider that it has been performed with a relatively low number of patients. In this regard, Newburger et $\mathrm{al}^{7}$ studied 280 patients, whereas Geva et al $^{5}$ analyzed 100 cases and Smythe et al ${ }^{1}$ studied 161 cases. These 3 important studies reached the same conclusion: clinical examination is the most useful method in the initial evaluation of children sent for heart murmur evaluation.

The present study documents our experience in 7 years of ambulatory care in public hospitals in Ribeirão Preto, SP, a city with a population of 500 million inhabitants. These patients are usually sent from 33 primary health care facilities, situated on the outskirts of town, to a central specialized unit previously mentioned ${ }^{2}$. Basically, we found the following 2 conflicting situations: children with an initial diagnosis of an innocent murmur whose cardiovascular abnormality was detected in complimentary tests, and patients with an initial diagnosis of cardiopathy that was not confirmed by the tests.

Group I-initial diagnosis of an innocent murmur and a final diagnosis of cardiopathy. Because of its high prevalence in healthy children, an innocent murmur is considered an important theme when discussing cardiopathy in children ${ }^{9-13}$. Although complementary tests, apart from electrocardiography, are not routinely performed in children with an initial diagnosis of an innocent murmur, the patients from this group had already undergone appropriate tests. In this situation, we found $17(0.7 \%)$ patients with an initial diagnosis of an innocent murmur and abnormal examinations. Although the individual analysis was taken into consideration ${ }^{14}$, we believe that this number reflects a very small incidence in a high-risk group, and these children would have been discharged if these tests had not been performed at that time. Additionally, examining these patients carefully, we found that 5 of them may be considered normal. Three children had mild mitral regurgitation, 1 had mild aortic regurgitation, exclusively diagnosed by Doppler echocardiography, and one had idiopathic dilation of the pulmonary artery trunk, which is usually considered a normal finding, in spite of the rare occurrence of complications ${ }^{15}$. It is important to point out that the patient with a history of rheumatic fever did not have aortic regurgitation according to the echocardiogram, which, nevertheless, may occur.

Excluding these 5 patients, 12 patients $(0.4 \%)$ had a diagnosis of cardiopathy. We stress that all these defects lead to small hemodynamic consequences for these patients, and lower long-term complications, apart from the case of dilated myocardial disease with an uncertain prognosis. In conclusion, if these groups of patients had their diagnosis established, based only on the initial diagnosis of innocent murmur, and had been immediately discharged, the risk of complications in the short- and medium- term follow-up would be very low but real, because defects like small VSD, MVP, small ASD, mild pulmonary stenosis, and asymptomatic Wolff-Parkinson-White syndrome are considered low-risk diseases.

Group 2-initial diagnosis of cardiopathy not confirmed by complementary tests. One hundred sixty-one (6.2\%) cases were detected, demonstrating that initially cardiopathy was excessively diagnosed. A detailed analysis of this group showed that the initial diagnosis of cardiopathy may have been modified if the patients had been reexamined. For example, patients initially suspected of having small VSD $(n=72)$ or subaortic stenosis $(n=10)$ corresponded to more than $50 \%$ of this group and represented a situation where the diagnosis was established based on the presence of innocent Still murmurs, which can, sometimes, be similar to that found in these 2 defects. Another situation frequently found is children with systolic murmurs in the pulmonary area. In 43 of our patients, ASD or mild pulmonary stenosis was suspected based on the presence of this murmur, which was not confirmed by complementary tests. We must remember that this murmur is the second most common type found in children and the possibility of misdiagnosis with ASD or mild pulmonary stenosis has already been reported ${ }^{12}$. An interesting aspect of our study was the presumed diagnosis of dilated myocardial disease based exclusively on X-ray findings that were not confirmed by echocardiography. The apparent cardiomegaly was attributed to inadequate examination techniques.

Despite all these considerations, it is clear that diagnosis of cardiopathy was excessively made in this group of patients, leading to further investigations that could have been avoided, and that could had had a physiological impact.

In conclusion, the possibility of misdiagnosis in the initial clinical evaluation of children referred to the hospital because of cardiac murmurs is real, reaching approximately $6 \%$ of cases, and, in our experience, the great majority of them are in patients with an initial diagnosis of cardiopathy not confirmed by complementary tests. The group with potentially deleterious complications (initial diagnosis of innocent murmur and abnormal examinations (group 1) was very small $(0.7 \%)$, and the diagnosed pathologies had small hemodynamic repercussion. It is also important to recognize the possibility of occasionally finding cardiopathy through complementary tests, as in 2 patients with Wolff-Parkinson-White syndrome, and 3 patients with innocent murmurs and mitral valve prolapse detected by echocardiography.

The costs of a diagnostic investigation should be considered, especially in our country and primarily in the public health services. We would like to conclude that an 
appropriate clinical cardiovascular evaluation might be an excellent instrument in the evaluation of children with a suspicion of cardiopathy ${ }^{16}$. Data here presented demonstrate, as already discussed ${ }^{17}$, that congenital cardiopathy tends to be excessively diagnosed, leading to the need for a careful evaluation before the diagnosis of a certain cardiopathy is reached. Immediate outhospital discharge of children with innocent murmurs seems to be an acceptable practice. Institutions involved in graduate and postgraduate studies must stress the importance of clinical examination in the evaluation of children suspected of having cardiopathy.

Criticisms of the study: the investigation is not original, because previous publications have already addressed the theme. However, the great number of patients and the absence of a similar study in our country make the investi- gation relevant, considering our social characteristics. The pragmatism exposed in clinical conduct, inherent to the results found, may be criticized; however, it is supported by the impossibility of performing echocardiography in all the children referred to a hospital with a suspicion of cardiopathy. In other centers, such conduct may be possible; however, the patients have already been evaluated. Despite the relative benignity of the cardiopathies found in group 1, the number of patients is small. A great number of patients in the study with an initial diagnosis of an innocent murmur did not undergo echocardiographic study, and that can potentially reveal a structural defect. Prospective echocardiographic investigation with a greater number of children with an initial diagnosis of an innocent murmur may strengthen the results and the suggested approach with these patients.

\section{References}

1. Smythe JF, Teixeira OHP, Vlad P, Demers PP, Feldman W. Initial evaluation of heart murmurs: are laboratory tests necessary? Pediatrics 1990; 86: 497-500.

2. Amaral FTV, Granzotti JA, Nunes MA. Avaliação cardiológica em crianças com suspeita de cardiopatia: resultados preliminares em 2000 pacientes. J Pediatria 1995; 71: 209-13

3. Amaral FTV, Granzotti JA, Nunes MA. Sopro cardíaco na criança. Experiência de um ambulatório especializado. Rev Paul Pediatr 1995; 13: 39-41.

4. Amaral F, Granzotti JA, Simões B. Perfil ambulatorial em cardiologia pediátrica na cidade de Ribeirão Preto. Resultados preliminares. Annals XXX Brazilian Congress of Pediatrics. Arq Bras Ped 1997(supl. 1); 27.

5. Geva T, Hegash J, Frand M. Reappraisal of the approach to the child with heart murmurs: is echocardiography mandatory? Int J Cardiol 1988; 19: 107-13.

6. Amaral FTV, Granzotti JA, Nunes MA. Abordagem da criança com sopro cardíaco. Importância diagnóstica dos exames complementares não invasivos. Arq Bras Cardiol 1995; 64: 195-9.

7. Newburger JM, Rosenthal A, Williams RG, Fellows K, Miettinen OS. Noninvasive tests in the initial evaluation of heart murmurs in children. N Engl J Med 1983; 308: 61-4

8. Danford DA, Nasir A, Gumbiner C. Cost assessment of the evaluation of heart murmurs in children. Pediatrics 1993; 91:365-8.
9. Thayer WS. Reflections of the interpretation of systolic cardiac murmurs. Am J Med Sci 1925; 169: 313-21

10. Gibson S. Clinical significance of heart murmurs in children. Med Clin North Am 1946; 30: 35-6.

11. Friedman S, Robie WA, Harris TN. Occurrence of innocent adventitious cardiac sounds in childhood. Pediatrics 1949; 4: 782-9.

12. Tavel M. The systolic murmur: innocent or guilty? Am J Cardiol 1977; 39: 757-9.

13. Amaral F. Sopros innocents em crianças. In: Porto CC, ed. Doenças do Coração. Prevenção e Tratamento, $1^{\mathrm{a}}$ ed. Guanabara-Koogan: Rio de Janeiro, 1998: 333-5.

14. Gidding SS, Rosenthal A. The interface between primary care and pediatric cardiology. Ped Clin North Am 1984; 31: 1367-88.

15. Andrews R, Colloby P, Hubner PJB. Pulmonary artery dissection in a patient with idiophatic dilatation of the pulmonary artery: a rare cause of sudden death Br Heart J 1993; 69: 268-9.

16. Amaral F. Diagnóstico em cardiologia pediátrica. Elementos clínicos não são obsoletos. Arq Bras Cardiol 1996; 67: 35-7.

17. Amaral F, Granzotti JA. Cardiologic evaluation of children with suspected heart disease: experience of a public outpatient clinic in Brazil. São Paulo Med J 1999; 117: 101-7. 